\section{Preparation of the Yeast Pichia pastoris for Transmission Electron Microscopy}

Benjamin A. Yount, Joan Lin-Cereghino,

Geoff P. Lin-Cereghino, and Marcia M. Fox

Dept. of Biological Sciences, U. of the Pacific, Stockton, CA mfoxx@pacific.edu

\section{Introduction}

The methylotropic yeast Pichia pastoris is a model organism for the study of autophagy ${ }^{1}$ and peroxisome biogenesis ${ }^{2}$. Being able to look at the organism via transmission electron microscopy (TEM) can yield valuable data on the morphology of the secretory pathway and many other organelles of interest ${ }^{3}$. However, preparing the yeast for TEM work can be very arduous and costly. One of the reasons $P$. pastoris is so hard to prepare for visualization is because its cell wall is very thick and tough compared to the membrane of a mammalian cell. Thus, P. pastoris is notoriously difficult to infiltrate with fixatives, a step necessary to maintain its ultrastructure 4 . This article outlines an efficient and cost effective way to prepare P. pastoris for TEM without the need for certain specialized equipment. With this protocol, excellent pictures can be obtained by using the buffers, $\mathrm{KMnO}_{4}$, sorbitol, and PIPES, along with glutaraldehyde. These components preserve the ultrastructure of the yeast without any apparent artifactual change in morphology 4

\section{Materials and Methods}

\section{Culturing procedure for $\boldsymbol{P}$. pastoris grown in YPD}

Inoculate $5 \mathrm{~mL}$ of YPD broth in a sterile $50 \mathrm{~mL}$ conical with $P$. pastoris colony grown from a plate, and grow overnight in a 30 degrees $\mathrm{C}$ shaker/incubator. Check the $\mathrm{OD}_{600}$ of the overnight culture, and then add the correct amount of the culture to 100 $\mathrm{mL}$ of the YPD (1\% Yeast extract, 2\% Peptone, 2\% Dextrose) in a $500 \mathrm{~mL}$ sterile baffled Erlenmeyer flask so that the concentration is $0.01 \mathrm{OD}_{600} / \mathrm{mL}$. Place the diluted culture in the shaker for approximately 12 hours. This will ensure that the culture will have an end $\mathrm{OD}_{600}$ reading between 0.5-1.0. Place yeast on ice before starting the fixation procedure.

\section{Culturing procedure for $\boldsymbol{P}$. pastoris grown in BMMY}

Inoculate $5 \mathrm{~mL}$ of YPD broth in a sterile $50 \mathrm{~mL}$ conical with P. pastoris colony grown from a plate, and grow overnight in a 30 degrees $\mathrm{C}$ shaker/incubator. Check the $\mathrm{OD}_{600}$ of the overnight culture. Calculate the amount of the culture to add to $100 \mathrm{~mL}$ of the BMMY (Buffered Methanol-complex Medium with 1\% Yeast extract, $0.5 \%$ methanol) so that the concentration is $0.012 \mathrm{OD}_{600}$ $/ \mathrm{mL}$. Pipette the exact amount of the overnight culture into a 1.5 $\mathrm{mL}$ microfuge tube. Centrifuge for 1 minute at 5,000 $\times \mathrm{g}$. Remove the supernatant, resuspend in $1 \mathrm{~mL}$ BMMY, and add the cells to the $500 \mathrm{~mL}$ sterile baffled Erlenmeyer flask containing $100 \mathrm{~mL}$ of BMMY. Place the diluted culture in the shaker for approximately 24 hours. This will ensure that the culture will have an end $\mathrm{OD}_{600}$ reading between 0.5-1.0. Place yeast on ice before starting the fixation procedure.

\section{Fixation:}

Using a procedure originally designed for S. cerevisiae, we have developed a fixation protocol to address the limitations of P. pastoris ${ }^{4}$. Measure $7.5 \mathrm{~mL}$ of culture into a $15 \mathrm{~mL}$ conical tube. Pour the culture into an equal volume of $2 \times$ fixative $(7.50 \mathrm{~mL} 0.4 \mathrm{M}$ PIPES buffer $+1.25 \mathrm{~mL} 2.4 \mathrm{M}$ sorbitol $+0.03 \mathrm{~mL} 1.0 \mathrm{M} \mathrm{MgCl}_{2}+$
$0.03 \mathrm{~mL} 1.0 \mathrm{M} \mathrm{CaCl}_{2}+7.50 \mathrm{~mL} 8 \%$ glutaraldehyde $+\mathrm{ddH}_{2} 0$ to 20 $\mathrm{mL})$. Incubate at room temperature ( 25 degrees $C$ ) for 5 minutes. Spin down cells at $1060 \times \mathrm{g}$ for 10 minutes, and decant supernatant. Resuspend the cells in $9.5 \mathrm{~mL}$ of $1 \mathrm{X}\left(1: 1 \mathrm{H}_{2} \mathrm{O}\right)$ fixative. Incubate overnight at 4 degrees $C$. Centrifuge cells at $1060 \times \mathrm{g}$ for 5 minutes, and then aspirate fixative. Resuspend the cells in $8 \mathrm{~mL}$ of $\mathrm{H}_{2} \mathrm{O}$ and incubate for 10 minutes. Centrifuge at $1060 \times \mathrm{g}$ for 5 minutes. Repeat water wash 3 times. For the last centrifugation, aspirate off the supernatant and resuspend the pellet in the tiny residual amount of water $(\sim 100 \mu \mathrm{L})$ that remains. Add $5 \mathrm{~mL}$ of $2 \% \mathrm{KMnO}_{4}$, and incubate 5 minutes at room temperature. Pellet cells at $1060 \times \mathrm{g}$ for 5 minutes. Aspirate fixative and overlay with fresh $2 \% \mathrm{KMnO}_{4}$. Incubate for 45 minutes at room temperature. Centrifuge cells at $1060 \times \mathrm{g}$ for 5 minutes. Aspirate permanganate solution, making sure not to disturb the pellet. Fill the $15 \mathrm{~mL}$ conical with $\mathrm{ddH}_{2} \mathrm{O}$, remove water, and repeat until no purple color is evident. Add $1 \%$ uranyl acetate and incubate at $4^{\circ} \mathrm{C}$ overnight. Remove the uranyl acetate with an aspirator. Add $5 \mathrm{~mL} \mathrm{H}_{2} \mathrm{O}$, mix, and centrifuge at $1060 \times$ g until pellet is settled. Repeat this step 3 times. Dehydrate in a graded series of ethanol from $25 \%-100 \% \mathrm{EtOH}$. Resuspend pellet in 2:1 EtOH : Spurr's resin $(3 \mathrm{~mL}: 1.5 \mathrm{~mL})$ in a $20 \mathrm{~mL}$ glass scintillation vial. Rotate at slow speed $(\sim 175 \mathrm{rpm})$ for 2 hours at room temperature. Remove the resin and replace with 1:1 EtOH : Spurr's resin $(1.5 \mathrm{~mL}: 1.5 \mathrm{~mL})$. Allow to rotate at slow speed uncapped overnight at room temperature. Remove the resin and add $2 \mathrm{~mL}$ of $100 \%$ resin. Rotate uncapped for 1 hour, and then remove resin after centrifugation by pipetting. Add $100 \%$ resin and rotate for 2 hours capped. Transfer the resin containing the cells into 1.5 $\mathrm{mL}$ microfuge tubes and centrifuge at $2000 \times \mathrm{g}$ for 10 minutes. Remove resin. Add 2 drops of pellet to each micromold with a Pasteur pipette and fill with additional $100 \%$ resin in micromolds. Place the micromolds in a 60 degrees $\mathrm{C}$ oven for 24 hours.

\section{Post-fixation:}

Trim the blocks to a $0.5 \mathrm{~mm}$ trapezoid face, and section. Use an Emcorp Diamond knife for sectioning at a speed of $1.6 \mathrm{~mm} / \mathrm{s}$ and at a thickness of 55-60 nm on a Leica Ultracut R microtome. Retrieve sections on 200 mesh copper grids, and capture the images on a computer. In our lab, images were captured using a Zeiss 109

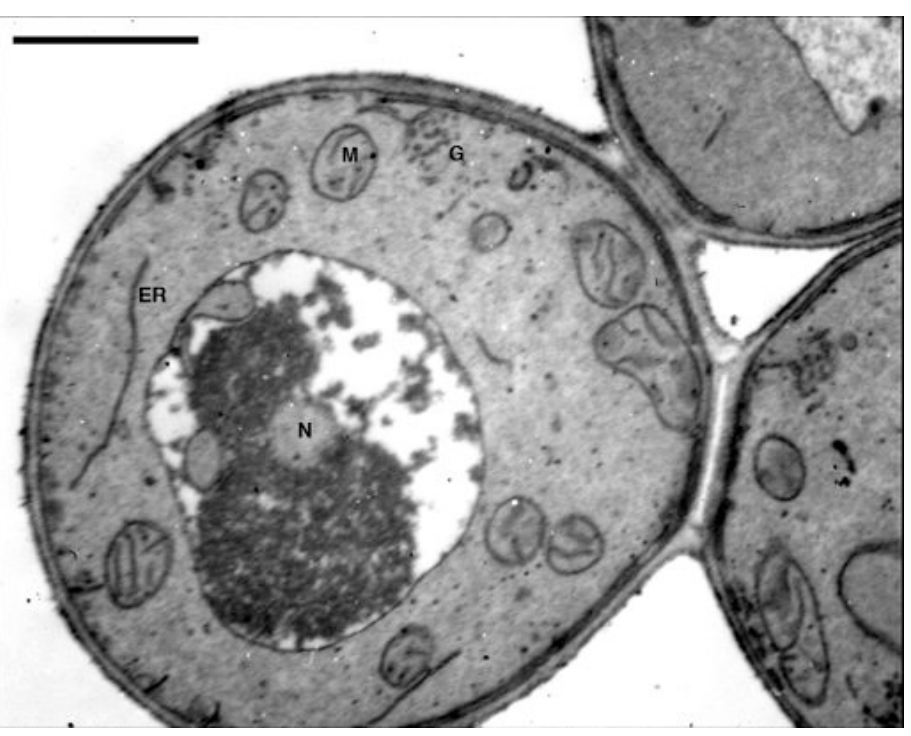

Figure 1: Electron micrograph of P. pastoris grown in YPD medium. $G=$ Golgi vesicles; $N=$ nucleus; $E R=$ endoplasmic reticulum; $M=$ mitochondria. Scale bar $=1 \mu \mathrm{m}$ 
TEM with a Micromax ccd digital camera (Princeton Instruments, Roper Scientific, Trenton, NJ), with the software program Winview (Princeton Instruments, Acton, Trenton, NJ).

\section{Results:}

This method allows researchers to look at various aspects of the morphology of $P$. pastoris. Structures commonly observed are: $\mathrm{mi}-$ tochondria, nuclei, vacuoles, peroxisomes (under methanol-growth conditions), endoplasmic reticuli, and Golgi (Figures 1 and 2). One of the most crucial aspects of working with P. pastoris for TEM work is the preparation of the cultures. The cell density should be between $0.5-1.0 \mathrm{OD}_{600}$. When there was an optical density greater than 1.0, the following problems occurred: a) budding scars were present on the cells; b) the cells were poorly infiltrated; c) when sectioning, the cells were ripped out of the resin because they cut at a different rate than the resin in which they were embedded; and d) there were many holes in the specimen so that when the electron beam came in contact with these holes, the sample was destroyed. In addition, the correct thickness of the sections is also important for capturing clear pictures. When the sections were too thick, sharp focus was extremely difficult, and when they were too thin, the sections disintegrated under the beam. When the samples had the optimal concentration of cells and the sections were the correct thickness, clear images were obtained.

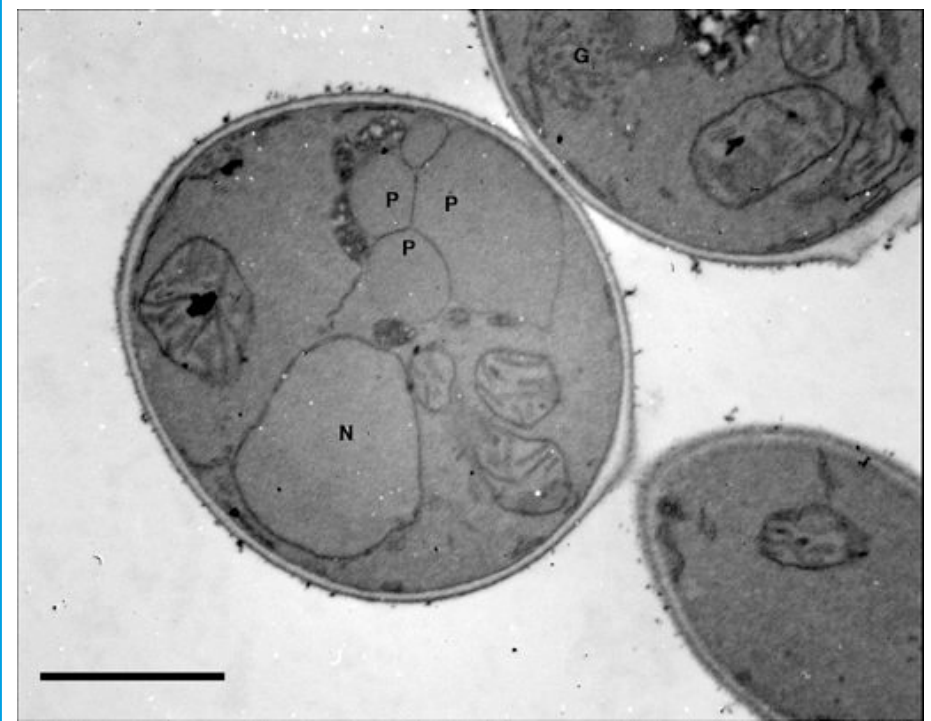

Figure 2: Electron micrograph of P. pastoris grown in BMMY medium. Methanol selectively induces the production of peroxisomes. $G=G o l g i$ vesicles; $N=$ nucleus; $E R=$ endoplasmic reticulum; $M=$ mitochondria; $P$ = peroxisome. Scale bar $=1 \mu \mathrm{m}$

\section{Conclusion:}

There are many possible research projects that can be carried out with the use of this protocol. For instance, organelle and cell structure can be investigated as a function of nutrition or the mutant background of a strain 6 . Although several methodologies are available for TEM of yeast, this protocol has been optimized specifically for $P$. pastoris. Starting with a culture inoculated with $P$. pastoris to ending with electron micrographs of the organism will take approximately 9 days using this protocol. Strict adherence to the protocol is essential. Skipping or changing one step can potentially ruin one's results. For instance, using formaldehyde in place of glutaraldehyde will cause poor fixation, leading to various problems described above. Using the traditional sodium cacodylate-osmium tetroxide fixation ${ }^{5}$ resulted in little to no fixation of $P$. pastoris. In addition, the use of counter stains, such as Reynold's lead citrate and uranyl acetate, is not necessary to increase the contrast of the samples because there was no apparent change in the pictures with or without these counter stains. Another attribute of this method is its minimal investment in advanced instrumentation, such as a speed vac or a specialized microwave oven. Thus, this method is particularly suitable for a research scientist with limited resources and time.

\section{Acknowledgements:}

This work was supported by NIH AREA (GM65882) grant to J.L-C. and G.P.L-C. This research is dedicated to the memory of the late Dr. Paul Richmond (University of the Pacific), who had a passion for microscopy and was the inspiration for this project. We are grateful to Dr. John Livesey (University of the Pacific) for his assistance with calculations

\section{References:}

1. Yuan, W., Stromhaug, P. E., \& Dunn Jr., W.A. (1999). Glucose-induced Autophagy of Peroxisomes in Pichia pastoris Requires a Unique E1-like Protein. Molecular Biology of the Cell, Vol. 10, 1353-1366.

2. Sakai, Y., Koller, A., Rangell, L.K., Keller, G.A., \& Subramani S. (1999). Pex19p Interacts with Pex3p and Pex10p and Is Essential for Peroxisome Biogenesis in Pichia pastoris. Molecular Biology of the Cell, Vol. 10, 1745-1761.

3. Soderholm, J., Bhattacharyya, D., Strongin, D., Markovitz, V., Connerly, P. L., Reinke, C. A., \& Glick, B. S. (2004). The Translational ER Localization Mechanism of Pichia pastoris Sec12. Developmental Cell, Vol. 6, 649-659.

4. Wright, Robin (2000). Transmission Electron Microscopy of Yeast. Microscopy Research and Technique, Vol. 51, 496-510.

5. Bozzola, J. J., \& Russell, L. D., Electron Microscopy $2^{\text {nd }}$ edition (1992). Jones and Bartlett Publishers, Sudbury, Pg. 23

6. Lin Cereghino, J. and J.M. Cregg. Heterologous protein expression in the methylotrophic yeast Pichia pastoris. 2000. FEMS Micro. Rev. 24: 45-66

\section{SERPETRENIX PHONE/FAX}

\section{Introducing the MaxView Plus Digital Camera Attachment System}

The MaxView Plus kit includes adapters to fit microscopes $\&$ optical devices with any of the following attachments; C-Mount, C/S-Mount, T-Mount, 23mm Eyepiece Port, $30 \mathrm{~mm}$ Eyepiece Port, and 1.25" Eyepiece Ports

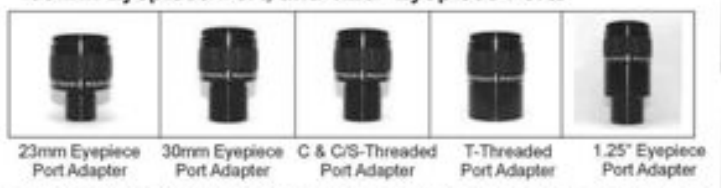

The MaxView Plus can also be used as a high quality wide angle eyepiece with the included thread-on eyeguard. The MaxView Plus is "T" threaded on top so you will need the appropriate Attachment Kit to mate it to your particular digtal camera. We carry dozens $\alpha$ attachment kits to fit most all popular digtal cameras and we update them regularly so your MaxView Plus will not become obsolete when it is time to upgrade to a new camera. If using it with a $35 \mathrm{~mm}$ SLR you will need the appropriate T-Ring for your brand of camera

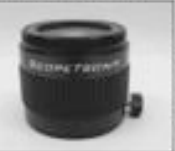

The heart of the MaxView system. A wide field $40 \mathrm{~mm}$ focal length four element symmetrical (Plossi) lens assembly specially mounted in a carefully designed custom cell. The lens is mounted only $1 \mathrm{~mm}$ from the top to maintain the close coupling required to reduce vignetting. The cell features a unique sliding collar assembly which serves several important functions, it allows quick and easy removal of the camera, it allows you to adjust eyeguard height for easy visual use, allows you to adjust magnification when used with a $35 \mathrm{~mm}$ camera, and allows you to adjust lens position to minimize vignetting with a digital camera.

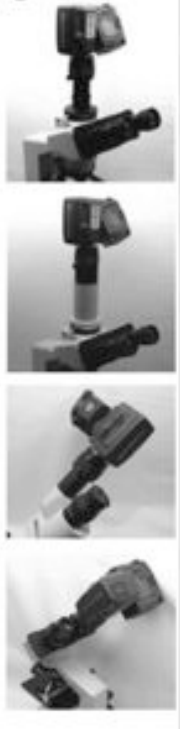

to minimize vignetting with a digital camera.
MaxView Plus Kit only $\$ 299.00$

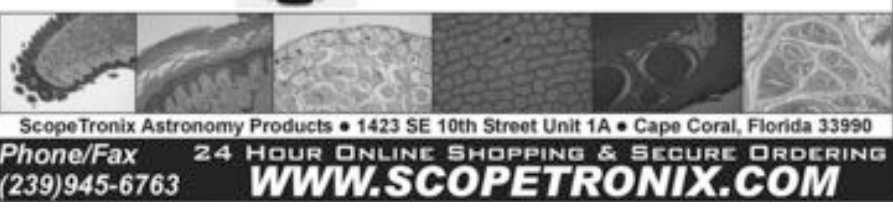

